\title{
Systematic Review of Radiological Analysis of Total Hip Replacement via Direct Anterior Approach in Comparison to Other Approaches - Study Protocol
}

Bartosz Maciąg ( $\sim$ bmaciag@wum.edu.pl)

Medical University of Warsaw https://orcid.org/0000-0002-0311-3735

Kuba Radzimowski

Medical University of Warsaw

Piotr Stępiński

Medical University of Warsaw

Jakub Szymczak

Medical University of Warsaw

Tomasz Albrewczyński

Medical University of Warsaw

Artur Stolarczyk

Medical University of Warsaw

Study protocol

Keywords: Total hip arthroplasty, Total hip replacement, THA, THR, direct anterior approach, DAA

Posted Date: October 12th, 2020

DOl: https://doi.org/10.21203/rs.3.rs-88637/v1

License: (1) This work is licensed under a Creative Commons Attribution 4.0 International License.

Read Full License 


\section{Abstract}

Background Total hip arthroplasty (THR) is considered as the most effective available method of treatment of end-stage hip osteoarthritis (OA). This surgery can be performed via many different approaches. One of the newly developed technique is called direct anterior approach (DAA). It gains popularity and recognition as the least traumatic to the soft tissues, thus quickening rehabilitation and improving joint stability in the early postoperative period. There are no systematic reviews covering this subject in terms of potential differences in radiological prosthesis placement, and while there is still unsatisfactory evidence concerning long-term outcomes of DAA, such review may prove useful in the debate. The objective of our study was to systematically collect and review available data from randomized-controlled trials (RCTs) regarding radiographic assessment of prosthesis placement after total hip arthroplasty using direct anterior approach compared to other common approaches.

Methods A systematic review of randomized controlled trials (RCTs) will be conducted. SciFinder, Scopus, ScienceDirect, PubMed, Embase, Clinical Key and The Cochrane Library databases will be searched without restriction to date up to June 2020. Primary outcomes will include measurements related with the radiological analysis of trials comparing use of DAA to other approaches used for THR, containing at least two of the following: femoral stem alignment, mean radiographic cup inclination, mean radiographic cup anteversion, mean radiographic cup abduction, position in Lewinnek's safe zone. Study selection will follow the Preferred Reporting Items for Systematic Reviews and Meta-Analyses guidelines, and the methodological appraisal of the studies will be assessed by the Cochrane Risk-of-Bias Tool for RCTs.

Discussion This systematic review will provide missing information regarding influence of differences between DAA and other surgical approaches in total hip replacement on prosthesis implants placement in case of femoral stem and cup. This may result in improving knowledge and awareness of surgeons, improving patients satisfaction and functional outcome and lowering the risk of hip dislocations and other complications.

Trial registration International Registration of Systematic reviews (PROSPERO) number CRD42019122675

\section{Background}

Total joint arthroplasty is considered the most effective way of treating the end-stage osteoarthritis (OA) of hip and knee joints $(1,2)$. It is estimated that every year more that one million total joint replacements are performed in Europe, while in Poland about 100000 annually. It is believed that our community is getting older and that is the reason why the number of total joint replacements will be growing, as well as patients expectations towards it. Even though total knee and hip replacement have wonderful outcome and are improving significantly patients, there is still place for improvement. This surgery can be performed via many different approaches. $(3,4)$ However, there is continuous dispute regarding the 
optimal choice of techniques, implants and approaches. The direct anterior approach (DAA) utilizes the intermuscular plane between sartorius and rectus muscles and tensor fascia lata, without the need to division of any tendon attachment of mentioned muscles. $(5,6)$. A direct anterior approach (DAA) gains popularity and recognition as the least traumatic to the soft tissues, thus probably quickening rehabilitation and improving joint stability in the early postoperative period and due to the shorter incision better cosmetic outcome. $(4,7-9)$ On the other side it is also considered to have steep learning curve and suspected to have higher risk of complications. (10) We believe that the difficulty of this approach might also have a negative effect on prosthesis placement, which could be assessed radiographically. It was previously proved, that proper prosthesis implants placement might be crucial for lowering the possibility of postoperative hip dislocations. $(11,12)$ Still, to our knowledge there are no systematic reviews covering this subject, and while there is still unsatisfactory evidence concerning long-term outcomes of DAA, such review may prove useful in the debate. There has been recently published meta-analysis of (13), in which authors analyzed outcomes of RCTs comparing DAA to other approaches in terms of postoperative analgesic requirements and pain scores, hospitalization time, length of incision, surgical time, complications such as: infection, wound healing problems, neurovascular damage, fractures, thrombosis, dislocations, component malpositioning, heterotopic ossification and death, but did not report analysis of radiological assessments in included studies. The objective of our study was to systematically collect and review available data regarding radiographic assessment of prosthesis placement after total hip arthroplasty using direct anterior approach compared to other common approaches.

\section{Methods}

\section{Protocol design and registration}

We will perform systematic review to summarize results from randomized controlled trials published with no restriction to date up to the June 2020. The development of this study protocol and the reporting of results will be performed accordingly to the Preferred Reporting Items for Systematic Reviews and Metaanalyses Protocol (PRISMA). (14) We used the PRISMA-P checklist when writing our report (15). This study protocol was previously registered with the International Registration of Systematic reviews (PROSPERO) and registration number CRD42019122675 was assigned.

(https://www.crd.york.ac.uk/prospero/display_record.php?RecordID=122675)

Such registration lowers the risk of duplicating systematic reviews and allows researchers to keep up with proper review process, resulting in minimizing of reporting bias.

\section{Search strategy}

We will conduct an English-language literature search of SciFinder, Scopus, ScienceDirect, PubMed, Embase, Clinical Key and The Cochrane Library without restriction to date up to June 2019.. The following search terms will be used: „total hip replacement," "total hip arthroplasty", "THA," "THR," "anterior," "direct anterior," "anterior supine intermuscular," "Hueter approach," and"Smith-Petersen." Search terms were combined using the Boolean operators "AND" or "OR." 


\section{Eligibility criteria}

We will include only randomized clinical trials involving patients over 18 years old (with primary hip osteoarthritis treated operatively), comparing DAA with other approaches and consisting of radiological analysis in at least of two of the following terms: femoral stem alignment, mean radiographic cup inclination, mean radiographic cup anteversion, mean radiographic cup abduction, position in Lewinnek's safe zone. We will exclude non-English studies, with full text not available, review or non-comparative studies, studies analyzing bilateral total hip replacement or hemiarthroplasty, and analyzing robotassisted surgeries.

\section{Data extraction (selection and coding)}

A summary of the participants, interventions, comparators and outcomes considered, as well as the type of studies included according to PICOS strategy, is provided in Table 1. (16)

Two independent researchers (BM, KR) will evaluate final set of studies in terms of: first author's name, country, publication year, type of surgical approach being compared to direct anteror approach, any significant differences at baseline, sample size, radiographic analysis. Revised Cochrane risk-of-bias tool for randomized trials (RoB 2) will be used (17). Disagreements between the review authors over the risk of bias in particular studies will be resolved by discussion, with involvement of a third review author (AS) where necessary. We will provide a narrative synthesis of the findings from the included studies, structured around the type of intervention, the target population characteristics, the type of outcome and the intervention content. Cohen's kappa coefficient will be calculated for interrater agreement between reviewers following assessment of study eligibility. (18) Kappa values $\leq 0$ will be interpreted as indicating no agreement, $0.01-0.20$ as none to slight, $0.21-0.40$ as fair, $0.41-0.60$ as moderate, $0.61-0.80$ as substantial, and $0.81-1.00$ as almost perfect agreement.

\section{Data synthesis}

We expect significant differences between studies in reporting radiological analysis with different values being assessed, therefore statistical data synthesis and exploration of consistency will probably be impossible to perform. However mean values of different prosthesis implant placement angles will be possible to analyzed between subgroups comparing DAA to single other approach.

\section{Discussion}

This systematic review will provide missing information regarding influence of differences between DAA and other surgical approaches in total hip replacement on prosthesis implants placement in case of femoral stem and cup. This may result in improving knowledge and awareness of surgeons, improving patients satisfaction and functional outcome and lowering the risk of hip dislocations and other complications. To the strengths of this study protocol belong the fact that it is the first systematic review of radiological analysis, comparing direct anterior approach to other used approaches for total hip replacement. It offers the highest level of evidence as only randomized controlled trials will be included. 
Direct anterior approach as more innovative and younger technique for performing total hip replacement might be described and analyzed in small number of randomized controlled trials.

\section{List Of Abbreviations}

THR - Total Hip Replacement

$\mathrm{OA}$ - osteoarthritis

DAA - Direct Anterior Approach

RCT - randomized-controlled trial

PROSPERO - International Registration of Systematic reviews

PRISMA - Preferred Reporting Items for Systematic Reviews and Meta-analyses Protocol

\section{Declarations}

\section{Ethics approval and consent to participate}

No institutional review board approval was required for this systematic review because the study included data that had been published previously. Results of this systematic review will be widely disseminated through publication in the valuable scientific peer-reviewed journal, concerning orthopaedic surgery.

\section{Consent for publication}

Not applicable

\section{Availability of data and materials}

The datasets used and/or analysed during the current study will be available from the corresponding author on reasonable request.

\section{Competing interests}

None declared.

\section{Funding}

The authors have not declared a specific grant for this research from any funding agency in the public, commercial or not-for-profit sectors.

\section{Authors' contributions}


$B M$ is the first and corresponding author; BM and AS conceived and designed the study, BM, KR, PS, JS, TW will acquire data; BM, KR, PS will analyze and interpret data; BM, KR and AS drafted the initial and final manuscripts, all authors performed critical revisions of the manuscript and approved the final version of the manuscript.

\section{Acknowledgements}

Not applicable

\section{Authors' information}

Corresponding author: BM E-mail: bmaciag@wum.edu.pl, Telephone: +48 881568144

Międzyleski Specialist Hospital, Str. Bursztynowa 2, 04-749 Warsaw, Poland

\section{Contact to authors:}

KR:kuba@radzimowski.com

PS: Piotr.stepinski01@gmail.com

JS: jakubszymczak92@gmail.com

TA: talbrewczynski@paleyeurope.com

AS: drstolarczyk@gmail.com

\section{References}

1. Jenkins PJ, Clement ND, Hamilton DF, Gaston P, Patton JT, Howie CR. Predicting the costeffectiveness of total hip and knee replacement: A health economic analysis. Bone Joint J. 2013;95B:115-21. doi: 10.1302/0301-620X.95B1.29835

2. Ethgen $O$, Bruye're $O$, Richy $F$, et al. Health-related quality of life in total hip and total knee arthroplasty. A qualitative and systematic review of the literature. J Bone Joint Surg Am. 2004;86A:963. doi: 2106/00004623-200405000-00012

3. Petis S, Howard JL, Lanting BL, et al. Surgical approach in primary total hip arthroplasty: anatomy, technique and clinical outcomes. Can J Surg. 2015;58:128-139. doi: 1503/cjs.007214

4. Moretti VM, Zachary DP. Surgical approaches for total hip arthroplasty. Indian J Orthop. 2017;51:368-76 doi: 10.4103/ortho.IJOrtho_317_16

5. Oinuma K, Eingartner C, Saito Y, et al. Total hip arthroplasty by a minimally invasive, direct anterior approach. Oper Orthop Traumatol. 2007;19:310. doi:1007/s00064-007-1209-3

6. Bender B, Nogler M, Hozack WJ. Direct anterior approach for total hip arthroplasty. Orthop Clin N Am 2009;40(3): 321e8. doi:1016/j.ocl.2009.01.003 
7. Cheng TE, Wallis JA, Taylor NF, Holden CT, Marks P, Smith CL, et al. A prospective randomized clinical trial in total hip arthroplasty-comparing early results between the direct anterior approach and the posterior approach. J Arthroplasty 2017;32(3):883e90. DOI: 10.1016/j.arth.2016.08.027

8. Zhao HY, Kang PD, Xia YY, Shi XJ, Nie Y, Pei FX. Comparison of early functional recovery after total hip arthroplasty using a direct anterior or posterolateral approach: a randomized controlled trial. J Arthroplasty 2017;32(11):3421e8. doi: 10.1016/j.arth.2017.05.056

9. Nistor DV, Caterev S, Bolboaca SD, Cosma D, Lucaciu DOG, Todor A. Transitioning to the direct anterior approach in total hip arthroplasty. Is it a true muscle sparing approach when performed by a low volume hip replacement surgeon? Int Orthop 2017;41(11):2245e52. doi: 10.1007/s00264-0173480-8

10. D'Arrigo C, Speranza A, Monaco E, Carcangiu A, Ferretti A. Learning curve in tissue sparing total hip replacement: comparison between different approaches. J Orthop Traumatol 2009;10(1):47e54. doi: 1007/s10195-008-0043-1

11. Abdel M. P., von Roth P., Jennings M. T., Hanssen A. D., Pagnano M. W. What safe zone? The vast majority of dislocated THAs are within the Lewinnek safe zone for acetabular component position. Clinical Orthopaedics and Related Research. 2016;474(2):386-391. doi: 10.1007/s11999015-4432-5

12. Murphy WS, Yun HH, Hayden B, Kowal JH, Murphy SB. The safe zone range for cup anteversion is narrower than for inclination in THA. Clin Orthop Relat Res. 2018;476(2):325-335. doi: 10.1007/s11999.0000000000000051.

13. Kucukdurmaz F, et al., A meta-analysis comparing the direct anterior with other approaches in primary total hip arthroplasty, The Surgeon (2018). doi:10.1016/j.surge.2018.09.001

14. Moher D, Liberati A, Tetzlaff J, Altman DG, The PRISMA Group (2009). Preferred Reporting Items for Systematic Reviews and Meta-Analyses:The PRISMA Statement. BMJ 2009;339:b2535, doi: 10.1136/bmj.b2535

15. Moher D, Shamseer L, Clarke M, Ghersi D, Liberati A, Petticrew M, Shekelle P, Stewart LA. Preferred Reporting Items for Systematic Review and Meta-Analysis Protocols (PRISMA-P) 2015 statement. Syst Rev. 2015;4(1):1. doi: 10.1186/2046-4053-4-1.

16. Higgins JPT, Green S, The Cochrane Collaboration. Chapter 5: Defining the review question and deveoloping criteria for including studies. In: Higgins JPT , Green S, eds. Cochrane handbook of systematic reviews. Version 5.0.1: The Cochrane Collaboration, 2008.

17. Sterne JAC, Savović J, Page MJ, Elbers RG, Blencowe NS, Boutron I, Cates CJ, Cheng H-Y, Corbett MS, Eldridge SM, Hernán MA, Hopewell S, Hróbjartsson A, Junqueira DR, Jüni P, Kirkham JJ, Lasserson T, Li T, McAleenan A, Reeves BC, Shepperd S, Shrier I, Stewart LA, Tilling K, White IR, Whiting PF, Higgins JPT. RoB 2: a revised tool for assessing risk of bias in randomised trials. BMJ, 2019 (in press)

18. Cohen, Jacob (1960). "A coefficient of agreement for nominal scales". Educational and Psychological Measurement. 20(1): 37-46. doi:1177/001316446002000104 


\section{Tables}

Due to technical limitations, table 1 is only available as a download in the Supplemental Files section.

\section{Supplementary Files}

This is a list of supplementary files associated with this preprint. Click to download.

- Table1.JPG

- PRISMAPchecklist11.doc 